\title{
Harmonic versus Chaos Controlled Oscillators in Hexapedal Locomotion
}

\author{
Luis A. Fuente ${ }^{1}$, Michael A. Lones ${ }^{2}$, Nigel T. Crook $^{1}$ and \\ Tjeerd V. Olde Scheper ${ }^{1}$ \\ 1 Department of Computing and Communication Technologies \\ \{lfuente-fernandez, ncrook, tvolde-scheper\}@brookes.ac.uk \\ Oxford Brookes University, Oxford OX3 0BP, England \\ 2 Department of Computer Science, M.Lones@hw.ac.uk \\ Heriot-Watt University, Edinburgh EH14 4AS, Scotland
}

\begin{abstract}
The behavioural diversity of chaotic oscillator can be controlled into periodic dynamics and used to model locomotion using central pattern generators. This paper shows how controlled chaotic oscillators may improve the adaptation of the robot locomotion behaviour to terrain uncertainties when compared to nonlinear harmonic oscillators. This is quantitatively assesses by the stability, changes of direction and steadiness of the robotic movements. Our results show that the controlled $\mathrm{Wu}$ oscillator promotes the emergence of adaptive locomotion when deterministic sensory feedback is used. They also suggest that the chaotic nature of chaos controlled oscillators increases the expressiveness of pattern generators to explore new locomotion gaits.
\end{abstract}

\section{Introduction}

Living organisms use distinctive locomotive abilities to interact adaptively with their environment. Central Pattern Generators (CPGs) are a popular approach inspired by the networks of neurons in spinal cords of vertebrates and invertebrate thoracic ganglia to develop accurate neuro-mechanical representations of locomotive behaviours in legged robots. Whilst traditional CPGs are often dynamically represented by coupled of harmonic oscillators $[1,2]$, recent studies envisage CPGs as a collection of coupled chaotic oscillators $[4,5]$ which mutually self-regulate according to environmental fluctuations and local influences.

Although the consideration of sensory information is not essential in the generation of synchronous pattern of motion, it increases the adaptability and robustness of control system in unsteady real environments. Orchestrating environmental signals to achieve efficient locomotion is a difficult task, specially when these signals come from potentially noise sources (i.e. robotic sensors). However, this is a task at which signalling networks are evidently good at solving in a biological contexts. We collectively refer to computational analogies of cellular signalling networks as Artificial Signalling Networks (ASNs) [8, 9].

In this article, we explore whether adaptability in multi-legged robots can be reinforced by the structural and dynamical properties of chaotic oscillators when 
compared to harmonic oscillators. Particularly, we aim to investigate whether the use of chaos controlled oscillators offer more flexibility in the generation of effective patterns of coordination with adequate sensory feedback loops. To do so, a two-layered architecture is used to map environmental signals into adaptive locomotive trajectories. The upper layer consists of a collection of interconnected ASNs. It receives real-time positional information and produces a set of environmental-based control directives, which collectively alter in a deterministic manner the dynamics of a CPG composed of either Hopf or controlled Wu oscillators in the bottom layer. Both oscillators exhibit a stable limit circle, but otherwise they lie at opposite ends of the dynamical spectrum: the former is a single steady state system and the latter shows ordered and chaotic behaviours depending on its governing parameters. We use an evolutionary algorithm to optimise computational analogies of signalling networks that, when stimulated with sensory feedback, tune the CPG's oscillatory trajectories appropriately. A simulated version of the T-Hex robot is used to evaluate the performance of both oscillators in a challenging environment.

The rest of the article is organised as follows: Section 2 introduces the oscillators addressed in this article, Section 3 describes the CPGs, Section 5 describes our methodology, Section 4 presents signalling networks, Section 6 presents results and Sec[8]tion 7 concludes.

\section{Central Pattern Generators}

Central Pattern Generators (CPGs) are a common way of modelling and generating locomotive gaits. Whilst they are often implemented using biologicallymotivated models such as feedforward neural networks [13] and artificial biochemical networks [12], they can also be considered as systems of coupled nonlinear oscillators. This kind of CPG favours distributed control approaches, leg synchronisation and the modulation of locomotion by simple control signals. Its simple structure also eases the integration of sensory information when CPGs made of coupled nonlinear oscillators are applied to the control of robotic locomotion.

\subsection{Hopf oscillator}

The Hopf oscillator (see Figure 1) is a single steady state dynamical system that exhibits harmonic oscillation [14]. It is defined by the following two differential equations:

$$
\dot{y}=\alpha\left(\mu-r^{2}\right) y-\omega z \quad \dot{z}=\beta\left(\mu-r^{2}\right) z-\omega y
$$

where $(y, z) \in \mathbb{R}^{2}$ are the state variables, $r=\sqrt{y^{2}+z^{2}}, A=\sqrt{\mu}$ is the oscillation amplitude, $\omega$ is the oscillation frequency and $\alpha$ and $\beta$ are positive constants that determine its convergence rate to the limit circle. From our perspective, the Hopf oscillator has three prominent benefits. First, it is able to generate smooth, stable and cyclic trajectories in the presence of small perturbations. Second, its output 
can be exclusively modulated by changing its frequency $\omega$ and amplitude $\mu$, whilst preserving the other parameters. This separation eases its optimisation by evolutionary algorithms [15]. Third, it eases the development of coupling terms in an analytical way.

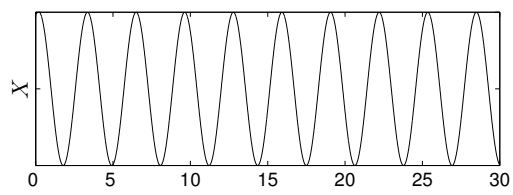

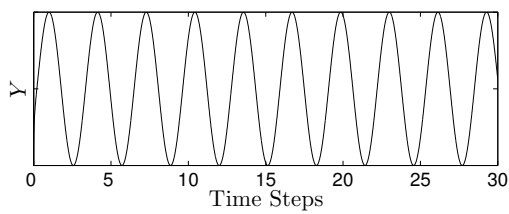

(a) Trajectories

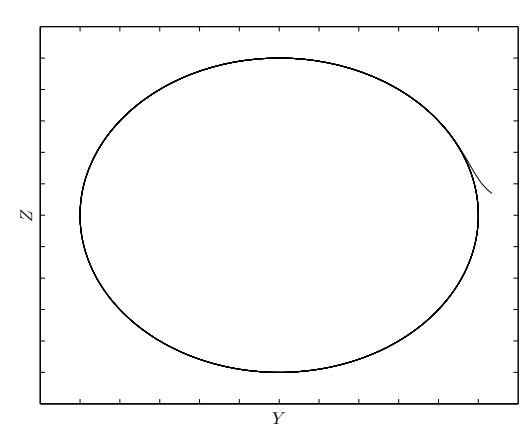

(b) Limit circle

Fig. 1: Outputs of the Hopf oscillator with $\mu=1, \omega=2, \alpha=5$ and $\beta=50$ and a phase portrait of its stable limit circle attractor.

\subsection{Wu oscillator}

The Wu oscillator (see Figures 2(a)-(b)) is a four-dimensional autonomous dynamical system able to exhibit a large variety of dynamical states [16]. It is defined by the following set of differential equations:

$$
\begin{aligned}
\dot{x} & =a(y-x)+e y z-k w & \dot{y} & =c x-d y-x z \\
\dot{z} & =x y-b z & \dot{w} & =r y+f y z
\end{aligned}
$$

where $(x, y, z, w) \in \mathbb{R}^{4}$ and $a, b, c, d, e, f, k$ and $r$ are all real constants. This oscillator is adopted for several reasons. Its four nonlinear terms help to rapidly propagate small alterations across its variables and, when coupled, throughout neighbouring oscillators. The $\mathrm{Wu}$ oscillator is also able to self-regulate and self-sustain its internal dynamics by adjusting its amplitude and frequency in response to external signals. When coupled, the system's overall state can only be deduced from the interactions amongst individual oscillators.

Trajectory Stabilisation Individual chaotic trajectories of the $\mathrm{Wu}$ oscillator are stabilised using the Rate Control of Chaos (RCC) method [17]. Unlike other chaos control strategies, the RRC method does not require any a priori knowledge about the presence of unstable periodic trajectories in a chaotic system. This approach relies on the expansion rate of an oscillator away from its trajectory to apply a small scale into its governing variables proportion to the 


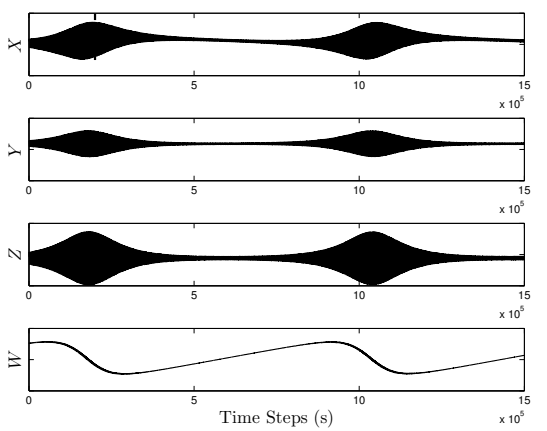

(a) Chaotic Wu trajectories
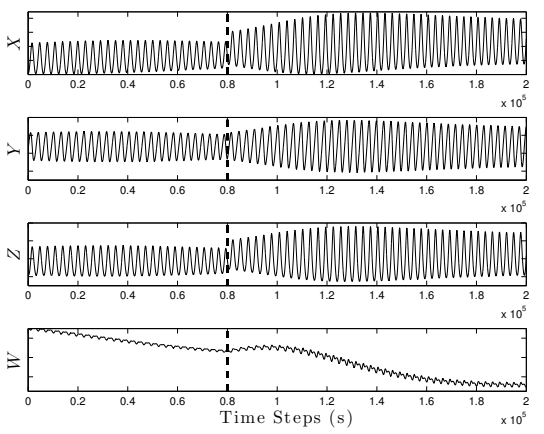

(c) Controlled Wu oscillator

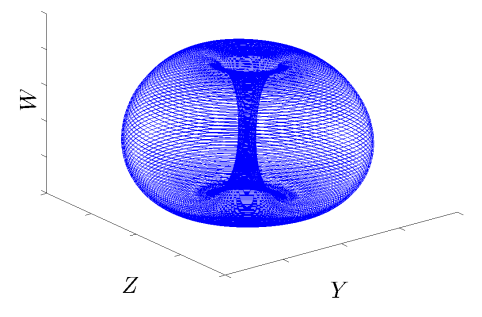

(b) Toroidal $\mathrm{Wu}$ attractor

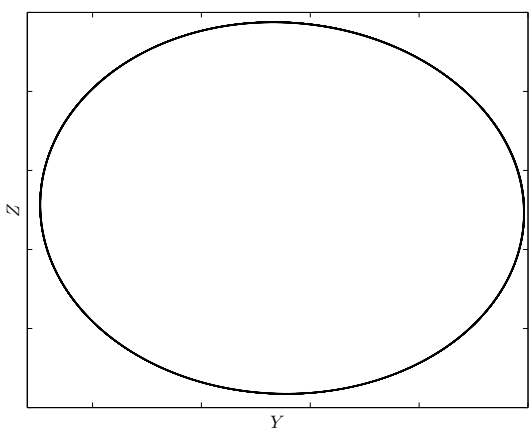

(d) Hopf/controlled Wu attractor

Fig. 2: Outputs of the Wu and controlled Wu oscillators. In both cases, the state parameters are $a=56, b=16, c=49, d=9, k=8, e=30, f=40$ and $r=-1943$. Using this configuration the Wu oscillator exhibits an aperiodic chaotic behaviour (a) with all its trajectories converging to a toroid attractor (b). When the RCC method is active at $t=0.8 \times 10^{4}$ (vertical dotted line), the oscillator's unstable and aperiodic trajectories (c) become harmonic with constant frequency and amplitude after an initial transient time (wandering period). (d) illustrates the stable limit circle attractor of the controlled Wu oscillator. 
divergence rate. Thus, it is possible to reduce the chaotic nature of the oscillator but preserving its chaotic properties. The extend of the perturbation can be calculated by determining the current proportion the variable occupies in its space. Within a robotic locomotion context, this also allows the modulation of the state of the robot based uniquely on the CPG's local influences and sensory feedback without any explicit knowledge of the robot's surrounding environment. The equations of the chaos controlled $\mathrm{Wu}$ oscillator are as follows:

$$
\begin{aligned}
\dot{x} & =a(y-x)+e \sigma_{x} y z-k w & \dot{y} & =c x-d y-\sigma_{y} x z \\
\dot{z} & =\sigma_{z} x y-b z & \dot{w} & =r y+f \sigma_{w} y z
\end{aligned}
$$

where $\sigma_{x}, \sigma_{y}, \sigma_{z}$ and $\sigma_{w}$ are the rate control functions and regulate the divergence rate between the variables in each of the nonlinear terms ${ }^{3}$. The controlled Wu oscillator is shown in Figures (see Figures 2(c)-(d)).

\section{Interlimb Coordination}

\subsection{Hopf Oscillator}

Interlimb coordination amongst Hopf oscillators is achieved by coupling them in a non-diffusive manner. It is non-diffusive in the sense that the influence amongst oscillator is constant over time. Contralateral and lateral adjacent oscillators are coupled through the $\dot{z}$ variable as follows:

$$
\dot{z}=\beta\left(\mu-r_{i}^{2}\right) z_{i}-\omega_{i} x_{i}+\sum k_{i j}\left(z_{i}+\lambda_{i j} z_{j}\right)
$$

where $i, j=1 \ldots 6$ are the oscillator indices, $k_{i j}$ is the diffusive coupling and $\lambda_{i j}$ is the coupling coefficient and establishes the phase relationships amongst coupled oscillators. The value of $\lambda$ is set to 1 if the oscillators excite each other and to -1 if the oscillators inhibit each other. We chose a coupling such that the tripod gait is stable. Figure 3(a) illustrates the coupled trajectories of the Hopf CPG.

\subsection{Chaos Controlled Wu Oscillator}

Chaos controlled $\mathrm{Wu}$ oscillators are coupled in a non-diffusive manner with time delay feedback. Contralateral and lateral adjacent oscillators are coupled through the $\dot{x}$ variable as follows:

$$
\dot{x}_{i}=a\left(y_{i}-x_{i}\right)+e \sigma\left(y_{i}, z_{i}\right) y_{i} z i-k w_{i}+\sum \tau_{i j}\left(x_{j}(t-\sigma)-x_{i}\right)
$$

where $i, j=1 \ldots 6$ are the oscillator indices, $\sigma$ is the time delay and $\tau_{i j}$ is the coupling coefficient and defines the the effect of the $i$ th on the $j$ th oscillator thereby establishing their phase relationship. The $y$ and $z$ trajectories of the controlled $\mathrm{Wu}$ oscillators are chosen to describe the motion of each leg because they increase stability when the robot moves on a flat surface. The coupling amongst trajectories also matches the tripod gait. Figure 3(b) illustrates the coupled trajectories of the Wu CPG.

\footnotetext{
${ }^{3}$ Refer to [17] for additional insight about controlling unstable trajectories using the
} RRC method. 

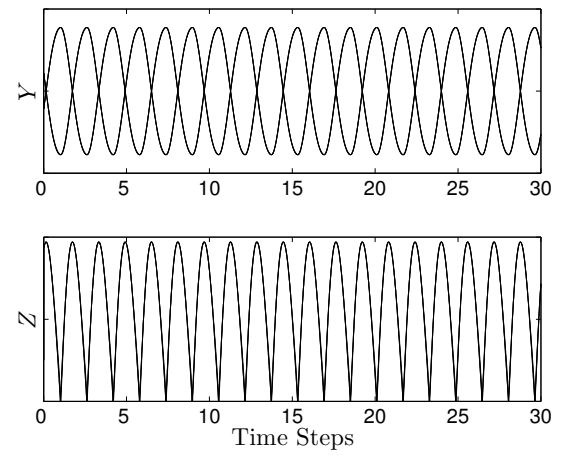

(a) Hopf trajectories
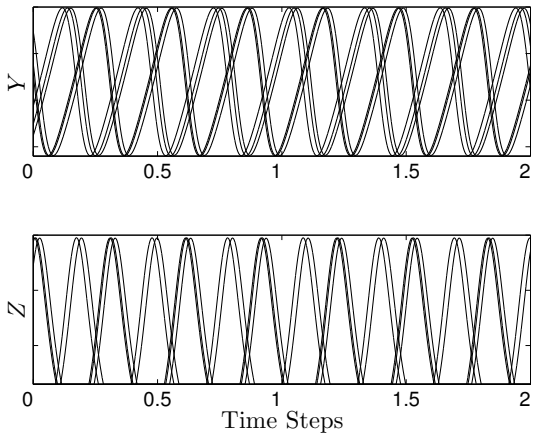

(b) Controlled Wu trajectories

Fig. 3: Coupled $y$ (upper plot) and $z$ (bottom plot) trajectories of two CPGs composed of six Hopf and controlled Wu oscillators. For the Hopf CPG, trajectories are obtained for $k_{i j}=1$. For the $\mathrm{Wu} \mathrm{CPG}$, trajectories are obtained using $\sigma=0.0118, \tau_{12}=1.0, \tau_{13}=1.8, \tau_{24}, \tau_{34}=\tau_{35}=1.1$, and $\tau_{56}=1.8$. We exploit the fact that $\tau_{i j}=\tau_{j i}$. Although $\mathrm{Wu}$ CPG trajectories are not completely synchronised, their minimal phase difference does not affect the performance of the control system and allows preserving stable forward locomotion.

\section{Coupled Artificial Signalling Network}

An Artificial signalling Network (ASN) is an enzyme-mediated abstraction of a cellular signalling process. Cellular signalling is the biological mechanism by which cells interact with other cells and their environment [7]. Broadly speaking, cellular signalling is a chain of events that, triggered by an extracellular signal, induces an adaptive cellular response. It begins with the binding of certain messengers and their later diffusion inside the cell. Such messengers then spread across the cell using signalling pathways until they reach the nucleus where they regulate gene expression and lead to the process by which a change in the cellular activity can be achieved.

Formally, an ASN is an indexed set of enzyme-analogous elements $E$ and a set of continuous-valued biochemical reactions. Each $e_{i} \in E$ has a set of substrates $s_{i}$, a product concentration $p_{i}$ and a regulatory function $f_{i}$. Substrate concentrations are mapped to product concentrations using the probabilistic Michaelis-Menten function which was previously shown to lead to the best performances when evolving ASNs capable of controlling trajectories in a prescribed manner [10]. The execution of the ASN starts with the random initialisation of the concentrations $\left(s_{i}\right.$ and $\left.p_{i}\right)$. External inputs are delivered to the network by the substrate concentration of nominated enzymes. At each time step, each enzyme $e_{i}$ applies its regulatory function $f_{i}$ to the current concentration of its substrates $s_{i}$ to determine the new concentration of its product $p_{i}$. After iterating the network a specific number of times $t_{S}$, the outputs are extracted from the final product concentration of designated enzymes. 
Biological responses to sensory information are often the result of the interaction of multiple pathways. Motivated by this observation, a number of authors have investigated the coupling of computational architectures based on models of interacting biochemical networks $[11,12]$. This article focuses on the interaction amongst signalling pathways since this is the principal process through which biological organisms handle environmental information. One of the main mechanisms that allows the exchange of information between interconnected signalling networks is crosstalk. In [8], we introduced a simple model of crosstalk within a connectionist architecture called a Coupled Artificial Signalling Network (CASN). Mimicking the structure of a signalling network, different types of external inputs are delivered to different sub-networks. These sub-networks (which are comparable to ASNs) do not have explicit interconnections, but they do contain crosstalk nodes which permit the exchange of information using a simple regulatory function. In this article, CASNs are optimised using evolutionary algorithms in order to transform sensory data into deterministic control directives that when applied to the governing parameters of each oscillator in the CPG alter its dynamics and elicit adaptive modifications in the locomotion pattern. From a locomotive perspective, coupling adjacent ASNs also favours the synchronisation of CASN-modulated oscillatory trajectories [8].

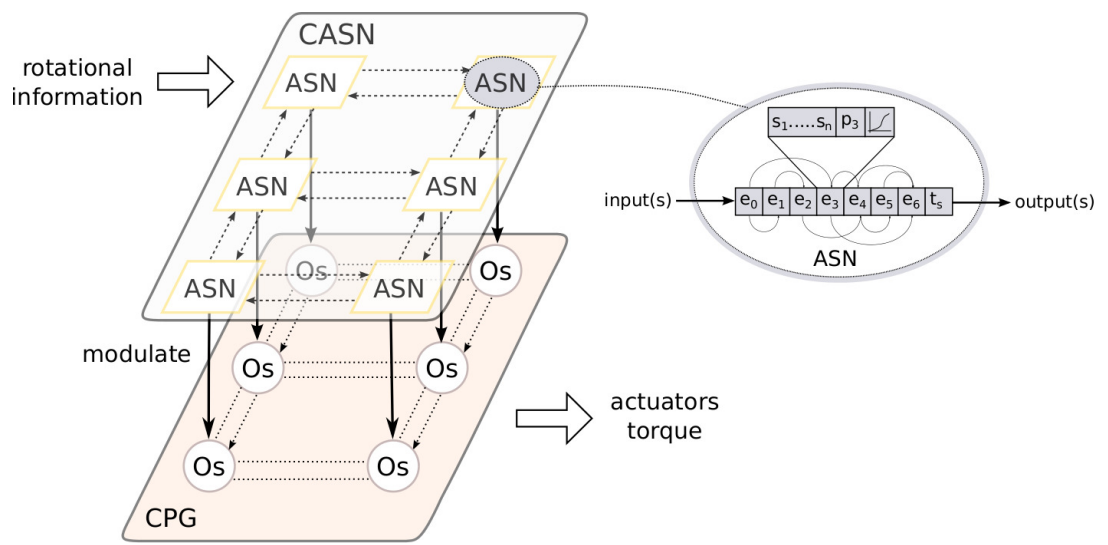

Fig. 4: Overview of the locomotive control system and coupling topology. Sensory feedback is delivered to an upper layer composed of six interconnected signalling networks, one per leg. Coupled networks comprise 10 enzyme-analogous elements. Gait trajectories are generated in the bottom layer, which contains a pattern generator made of six nonlinear oscillators. This layer, whose connectivity mirrors the upper layer, receives control directives that modulate the oscillatory trajectories, also one per leg. Finally, these are transformed into actuator positions. 


\section{Controlling Legged Robot Locomotion}

A simulated model (see Figure 5) of the commercial T-Hex robot is used to evaluate the expressiveness of the Hopf and the Wu CPGs. The T-Hex is a 24DoF hexapedal robot manufactured by Lynxmotion [18]. It has four joints per leg connected by actuators at the corners. The robot initially walks using the tripod gait, which is described by the moving of three legs simultaneously in each step. Its limited adaptability on irregular surfaces is exploited to determine the capacity of the CPGs to deliver reactive locomotion using local sensory feedback. The robot is simulated using the Open Dynamics (ODE) physics engine with a step side of $\Delta_{t}=0.01 \mathrm{~s}$, friction of $200 \mathrm{~N}$, CFM (an ODE parameter) of $10^{-5}$ and standard gravity. Actuators have a maximum angular velocity of $4 \mathrm{~ms}^{-1}$ and a maximum torque of $70 \mathrm{Nm}$. Their movements are limited in both the z-axis plane for the coxa joint and the $\mathrm{x}$-axis for the femur, tibia and tarsal joints, to a maximum rotation of $90^{\circ}$ and a minimum rotation of $-90^{\circ}$. These values are sufficient to simulate the characteristics of the physical T-Hex $[3,10]$. The CASN is executed every 20 simulation steps.

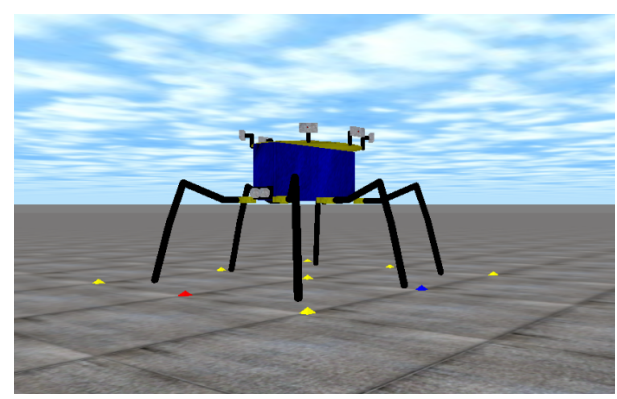

Fig. 5: Simulated T-Hex robot in Open Dynamics Engine.

\subsection{Gait Generation}

The task is to evolve a CASN capable of generating control signals that when applied to the oscillators' governing parameters originate different outputs in the CPG, i.e patterns of coordination which would cause the robot to displace away from its starting point. The aim is to measure qualitatively whether chaotic properties of the controlled $\mathrm{Wu}$ oscillators are sufficient to generate adaptive patterns of movement in response to deterministic sensory feedback when compared with the harmonic Hopf oscillator. The movement of each leg is controlled. The CASN consists of six individual ASN uniquely coupled to its adjacent ASN using a fixed crosstalk rate of 0.5. Each ASN is immediately connected with its corresponding bottom-level oscillator, whose output dictates the gait trajectory its matching leg. The controller fitness is the Euclidean distance minus the 
lateral displacement walked by the robot within an evaluation period of 4000 simulation steps. Both the Hopf and the Wu CPGs are randomly initialised and numerically integrated using the fifth-order Dormand-Prince method with step sizes of $\Delta_{t_{H}}=0.01$ and $\Delta_{t_{W}}=0.00001$ respectively. The selected $y$ and $z$ trajectories are scaled to a maximum height of $40 \mathrm{~mm}$ and a maximum length of $30 \mathrm{~mm}$ respectively, and sampled with a rate of $s_{r}=\pi / 4\left(\approx 40 t_{H}\right.$ and $\left.\approx 380 t_{W}\right)$. The population size is 200 , with a generation limit of 100 .

The rotational readings along the three Cartesian axes of each leg with respect to the centre of the robot represent the inputs of each ASN. They are the easiest feedback that gives actual insight into the robot stability and terrain features. Our objective is to calculate the rate of control needed to stabilise the robot whilst promoting the emergence of different patterns of movement. Rotational values are matched to the $[-\pi / 3, \pi / 3]$ interval, linearly scaled to the concentration range of $[0,1]$, and delivered to the ASN through its substrate concentrations. Values out of the rotation interval indicate that the robot has fallen. For the Hopf CPG, each ASN has two outputs which match the $\omega$ and $\mu$ Hopf parameters and modulate them in the intervals $[0,4]$ and $[0,8]$ respectively. For the $\mathrm{Wu} \mathrm{CPG}$, each ASN has also two outputs which match the $c$ and $b \mathrm{Wu}$ constant and modulate them in the intervals $[44,54]$ and $[11,21]$ respectively. In both cases, the outputs are in the range of $[0,1]$. The behaviour of the Hopf and controlled $\mathrm{Wu}$ pattern generators is evaluated on an uneven terrain consisting of a starting zone for the robot and a randomly generated uneven terrain, which comprises a mesh of 500 boxes with randomly chosen heights. Each box has a side of $20 \mathrm{~mm}$ and incremental height between $20 \mathrm{~mm}$ and $45 \mathrm{~mm}$. Values over these thresholds prevent the robot from moving forwards.

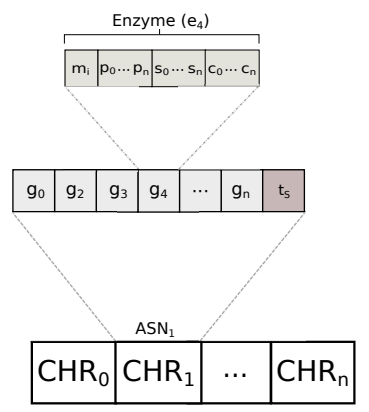

Fig. 6: Linear encoding of the CASN network used by the evolutionary algorithm, also showing how individual ASN and enzymes are represented. 


\subsection{Evolving Coupled Artificial Signalling Networks}

CASNs are evolved using a standard generational evolutionary algorithm with tournament selection $($ size $=4$ ), uniform crossover (rate $=0.3$ ), and point mutation (rate $=0.05)$. Each individual in the population is encoded as an indexed sequence of chromosomes, each of which represents an ASN (see Figure 6). A multi-chromosomal representation favours the evolution of problems with growing complexity and increases modularity [19]. Signalling networks are encoded as a set of 10 indexed genes followed by timing information. Crossover points lie between gene boundaries and chromosome shuffling is not permitted. Inputs and outputs $\left(s_{i}\right.$ and $\left.p_{i}\right)$ are represented by their absolute indices. Mutation is restricted to the set of operations in [20] to embrace biochemical plausibility in the evolution of enzymatic graphs. Chemical concentrations and function parameters are represented using floating-point values and mutated using a Gaussian function centred around the current value.

\section{Results}

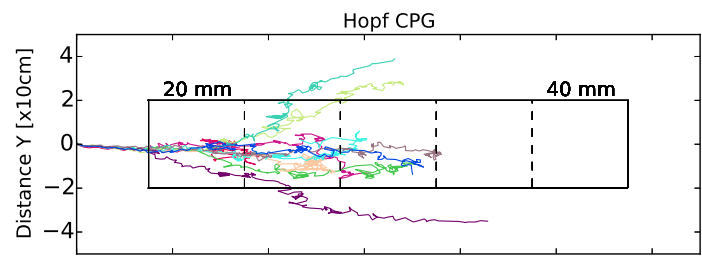

Wu CPG

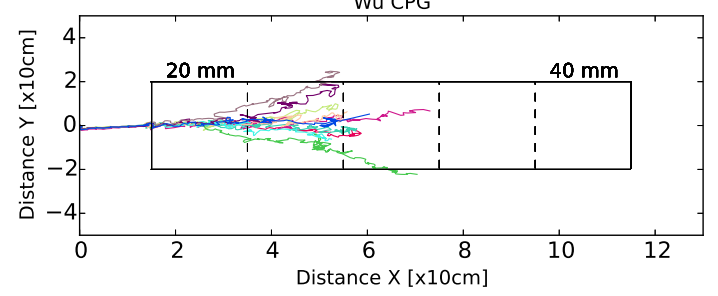

Fig. 7: Trajectories of each of the 10 evolved robot controllers when moving forwards in the test arena. The box represented with a solid line illustrates the bounds of the uneven terrain. Its initial maximum height is $20 \mathrm{~mm}$ and increases in steps of $5 \mathrm{~mm}$ after each horizontal dashed line up to $40 \mathrm{~mm}$.

The results show that CASNs can be used to deliver reactive control directives to CPGs with distinctive dynamics. While the robots actuated by the Hopf CPG exhibits an average walked distance of $67 \mathrm{~cm}$ (s.d. $21 \mathrm{~cm})$, the robots actuated by the controlled $\mathrm{Wu}$ CPG exhibit an averaged walked distance of $58 \mathrm{~cm}$ (s.d. $12 \mathrm{~cm}$ ). However, the performance of the Hopf CPG decreases drastically when the robot steps over the uneven terrain. Figure 7 shows the trajectories of each robot controlled during the evaluation time. Notably, the trajectories of the 
Hopf GPG start diverging apart as the robot steps over the test area. The results is that, in some runs, the robot laterally exits the uneven section of the terrain, giving rise to the furthest walking distances. Particularly, Hopf CPGbased robots experience noticeable difficulties to move when the terrain's height is $20 \mathrm{~mm}$ and nearly do not move when the terrain's height is up to $25 \mathrm{~mm}$. On the contrary, the trajectories of the Wu CPG remain grouped until the terrain's height is approximately $25 \mathrm{~mm}$, the movement at which they progressively diverge always within the test arena. This may suggests that the controlled Wu CPGs exhibit a better regulatory capacity as the intensity of the sensory feedback increases.

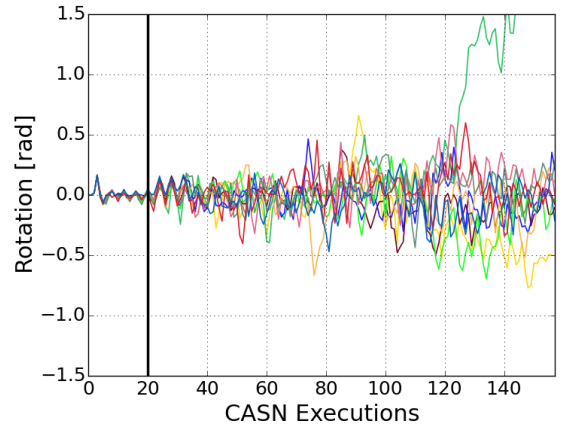

(a) $x$-axis, Hopf CPG

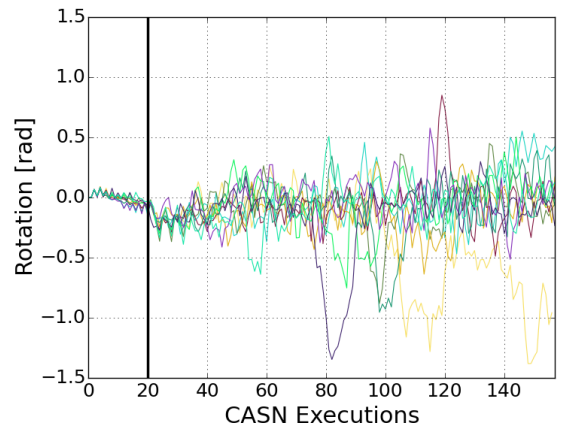

(c) $y$-axis, Hopf CPG

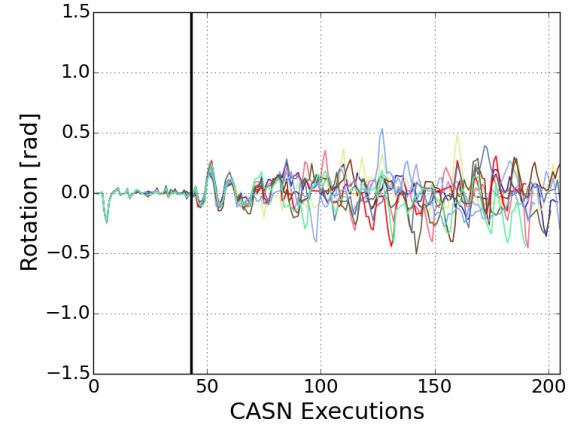

(b) $x$-axis, Wu CPG

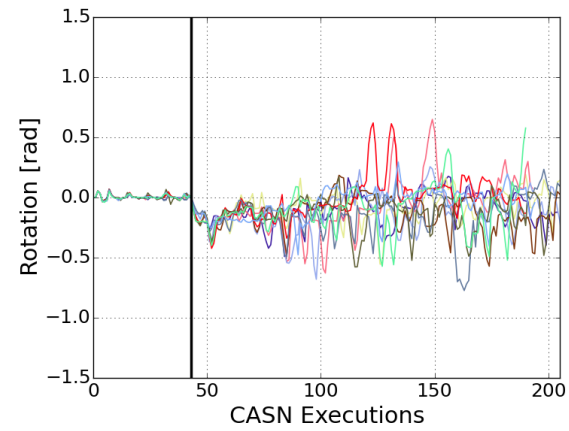

(d) $y$-axis, Wu CPG

Fig. 8: Rotations of the robot body along the $x$ - and $y$-axes for the Hopf CPGs (left plot) and the controlled $\mathrm{Wu}$ CPGs (right plot) every time the CASN is executed during the evaluation period. The vertical solid black line indiate the movement at which the robot reaches the uneven terrain.

Figure 8 illustrates the overall rotation of the robot body along the $x$-axis (a)(b) and $y$-axis (c)-(d) for both CPGs. Attending to the number CASN executions, it is also noticeable that robots actuated by the Hopf CPG reach the uneven part of the terrain quicker than the ones actuated by the controlled Wu CPG. 
However, the controlled $\mathrm{Wu}$ CPGs lead to more stable and steady locomotive movements on the flat surface despite that the $\mathrm{Wu}$ oscillators are not perfectly synchronised. A possible explanation is that the coupling error may promote stability since representative sensory feedback is received to the CASN from the starting of the evaluation period. Further, it is also evident that the Hopf CPGs readily produce sharp and unbalanced rotational trajectories. As can be seen in Figure 8(c) the simulated robot is slight tilted towards the right throughout the evaluation time. Interestingly, the robots actuated by Hopf CPGs have less difficulty stepping over the rough terrains, but they exhibit more fuzzy rotational trajectories as the complexity of the terrain increases. The robots actuated by the controlled Wu CPG behave in an opposite manner. They show abrupt changes in their walking direction while stepping over the uneven terrain, but they manage to control their stability while walking over it. We can hypothesise that this is a consequence of the flexibility of chaos controlled $\mathrm{Wu}$ oscillator to explore new pattern of coordination which appears to enhance the stabilisation of the robot in different terrains.
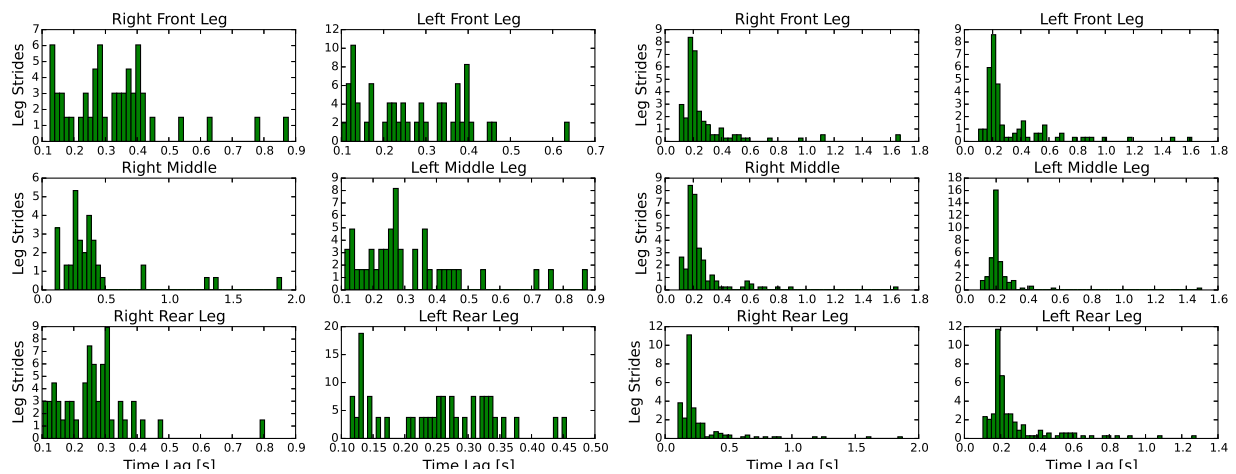

(a) Hopf CPG

(b) Controlled $\mathrm{Wu} \mathrm{CPG}$

Fig. 9: Example of the distributions of the time interval between consecutive touchdowns for each leg of the simulated robot when actuated by a Hopf CPG (left plot) and a controlled Wu CPG (right plot) during the evaluation time.

Adaptive locomotion is inherently difficult to analyse. This is because locomotive patterns of coordination not only depend on sensory feedback but also on the local interactions amongst the oscillators in a CPG. However, techniques to study the temporal distribution of the stepping patterns can be used to provide some insight into the behaviour of the CPG [21]. Figure 9 exemplifies the patterns of movement of two runs as the distribution of the time interval between consecutive touchdowns for each leg of the hexapod robot. In general, we found that the $\mathrm{Wu}$ CPGs have a clearly defined stepping pattern, which suggests that some self-induced patterns of motion may exist for this type of controller.

Figure 9(a) depicts histograms whose shape resembles a Gaussian-like distribution with a time lag mean steadily centred at $0.2 \mathrm{~s}$. This is indicative that the 
robot changes its initial fast tripod gait to another locomotive gait with smaller stance phase and longer swinging phase. This is perhaps surprising since there is not explicit coordination between oscillators during the evaluation time apart form the initial tripod coupling. In addition, this also suggests that anti-phase synchronisation between legs arises despite the dissimilarity of the sensory feedback. To a certain extent, this is also a consequence of the RCC method which regulates the effect that external perturbations have in the controlled Wu oscillator by allowing it to migrate to another area in the state space whilst preserving the integrity of its limit cycle. On the contrary, the Hopf CPGs produce rather irregular patterns of coordination (see Figure $9(\mathrm{~b})$ in which no real synchronisation occurs amongst oscillators. As a consequence, the robot either preserves its initial tripod coupling while exploring gaits around it, or explores new patterns of coordination but it is unable to preserve any of them. Nonetheless, the Hopf CPG is sufficient to induce reactive locomotive patterns when the irregularities of the terrain are not severe. This also explains the differences in performance seen in Figures 7 and 8, with the controlled Wu CPGs leading to more stable solutions whose patterns of coordination are more likely to adapt to changing environments.

\section{Conclusions}

In this paper, we have compared the performance of two different CPGs based on the Hopf and $\mathrm{Wu}$ systems in a rough terrain with incremental difficulty. Sensory information was added to the systems using CASNs, which were optimised for each controller using evolutionary algorithms.

The robots actuated by the Hopf CPG achieve the furthest walking distances and show the fastest locomotive paces, but also exhibit a remarkable lack of stability on rough terrains. The robots actuated by the controlled Wu CPG exhibit more deterministic patterns of locomotion. Likewise, the intrinsic nature of the controlled $\mathrm{Wu}$ oscillator allows the emergence of new patterns of adaptation in a reactive and efficient manner and increases the flexibility of CPGs to explore different patterns of motion when compared with the Hopf oscillator. Overall, it appears that the chaotic nature of the chaotic rate controlled Wu CPG enhances the development of adaptive and robust behaviours using sensory feedback.

In future work, we plan to investigate the importance of sensory feedback in the generation of differential patterns of motion and to evaluate the performance of both CPGs in the physical T-Hex and alternative surfaces.

\section{References}

1. Pina Filho de, A. C., Dutra, S. M., Raptopoulos, L. S. C: Modelling of a bipedal robot using mutually coupled oscillators Biological Cybernetics, 92, 1, 1-7 (2005)

2. Righetti, L., Ijspeert, A. J.: Design methodologies for central pattern generators: an application to crawling humanoids Proceedings of Robotics: Science and Systems, 191-198 (2006). 
3. Fuente, L. A., Lones, M. A., Turner, A. P., Caves, L. S., Stepney S., Tyrrell A. M.: Adaptive robotic gait control using coupled artificial signalling networks, hopf oscillators and inverse kinematics. 15th IEEE Congress on Evolutionary Computation, 1435-1442 (2013)

4. Kuniyoshi, Y., Suzuki, S.: Dynamic emergence and adaptation of behaviour through embodiment as coupled chaotic fields IEEE/RSJ International conference on Intelligent Robots and Systems, 2, 2042-2049 (2004)

5. Matthey, L., Righetti, L., Ijspeert, A. J.: Experimental study of limit cycle and chaotic controllers for the locomotion of centipede robots IEEE/RSJ International Conference on Intelligent Robots and Systems, 1860-1865 (2008).

6. Fasca, M., Arena, P., Fortuna, L.: Bio-inspired emergent control of locomotion systems World Scientific series of Nonlinear Science, A, 48 (2004)

7. Cell Signalling: $\mathrm{H}_{2} \mathrm{O}_{2}$ a necessary evil for cell signalling, Sience, 312, 1882-1883 (2006)

8. Fuente, L. A., Lones, M. A., Turner, A. P., Caves, L. S., Stepney S., Tyrrell A. M.: Evolved artificial Signalling networks for the Control of Conservative Complex Dynamical Systems The 9th Information Processing in Cells and Tissues, 7223, 38-49 (2002)

9. Fuente, L. A., Lones, M. A., Turner, A. P., Caves, L. S., Stepney S., Tyrrell A. M.: Computational models of signalling networks for non-linear control BioSystems, 122, 2, 122-130 (2013)

10. Fuente, L. A., A decentralised control architecture: coupled artificial signalling networks, PhD Thesis (2014)

11. Lones, M. A., Caves, L. S., Stepney S., Tyrrell A. M.: Controlling legged Robots with coupled artificial biochemical networks ECAL 2011, MIT Press, 465-472 (2011)

12. Lones, M. A., Fuente, L. A., Turner, A. P., Caves, L. S., Stepney S., Tyrrell A. M.: Artificial Biochemical Networks: Evolving dynamical systems to control dynamical systems IEEE Transaction on Evolutionary Computation, 18, 2, 145-166 (2014).

13. Moioli R. C., Vargas, P. A., Husbands, P.: A multiple hormone approach to homeostatic control of conflicting behaviours in an autonomous mobile robot IEEE 11th Congress on Evolutionary Computation, 47-54, (2009)

14. Righetti, L., Ijspeert, A. J.: Pattern generators with sensory feedback for the control of quadruped locomotion IEEE International Conference on Robotic and Automation, 819-824 (2008)

15. Wright, J., Jordanov, I.: Intelligent Approaches in Locomotion The 2012 International Joint Conference in Neural Networks (IJCNN 2012) pp. 1-8 (2012)

16. Wu, W., Zengqiang, C., Zhuzhi, Y.: The evolution of a novel four-dimensional autonomous system: among 3-torus, limit cycle, 2-torus, chaos and hyper-chaos Chaos, Solitions and Fractals, 39, 5, 2340-2356 (2009)

17. Scheper, T. O.: Why metabolic systems are rarely chaotic? BioSystems, 94, 1-2, 145-152 (2008)

18. Lynxmotion Ltd, 4-dof t-hex combo kit for bot board/ssc-32/bap28, Available at: http://www.lynxmotion.com/c-151-t-hex-4-dof.aspx.

19. Mayer, H. A., Spitzlinger, M.: Multi-chromosomal representations and chromosome shuffling in evolutionary algorithms The 2003 Congress on Evolutionary Computation, 2, 1145-1149 (2003)

20. Ziegler, J., Banzhaf, W.: Evolving control metabolisms for a robot Artificial Life, 7, 171-190 (2001)

21. Abourachid, A.; Herbin, A., Hacker, R., Maes, L.: Experimental study of coordination patterns during unsteady locomotion in mammals Journal of Experimental Biology, 210, 366-372 (2007) 